\title{
Gore-Tex Medialisation Thyroplasty for Unilateral Vocal Cord Palsy: A Tertiary Centre 7 Years Experience
}

\author{
Dayangku NPS ${ }^{\mathrm{a}}$, Marina $\mathrm{MB}^{\mathrm{a}}$, Mawaddah $\mathrm{A}^{\mathrm{a}}$, Sharifa Ezat WP ${ }^{\mathrm{b}}$, Abdullah $\mathrm{S}^{\mathrm{a}}$

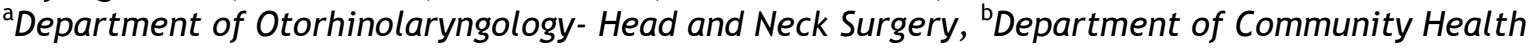 \\ Universiti Kebangsaan Malaysia Medical Centre. Jalan Yaacob Latiff, Cheras 56000 Kuala Lumpur, Malaysia.
}

\begin{abstract}
Background: The resultant dysphonia and aspiration in unilateral vocal cord palsy can be overcome with medialisation thyroplasty. With this background, we aim to determine the aetiology of the unilateral vocal cord palsy and effectiveness of the phonosurgical procedure with Gore-Tex as a sole treatment. Methods: Within a seven year period, 37 Gore-Tex medialisation thyroplasty were performed for unilateral vocal cord palsy at our institution and medical records were retrospectively reviewed. Results: There were 18 males and 19 females with mean age of 48.7 years (range 19-81 years). The predominant aetiology was thyroidectomy $(43.2 \%)$ with benign thyroid disease predominates $(n=13)$ over thyroid malignancy $(n=3)$. Voice outcome was evaluated subjectively using visual analogue scoring system, results indicating that Gore-Tex medialisation thyroplasty was effective in addressing dysphonia in $62.5 \%(n=15)$ patients. However it alone cannot address aspiration seen in those with high vagal nerve lesion. Airway compromise occurred in two cases postoperatively (5.4\%) presenting as acute stridor. Conclusion: In unilateral vocal cord palsy, Gore-Tex medialisation thyroplasty can effectively improve the resultant dysphonia and often accompanying aspiration which would otherwise be disabling for the patients.
\end{abstract}

KEYWORDS: Gore-Tex medialisation thyroplasty, vocal cord paralysis, thyroidectomy, voice outcome

\section{INTRODUCTION}

Vocal cord paralysis results in incomplete glottal closure which will lead to poor voice quality and diminished glottal competency with aspiration. Isshiki type 1 medialisation thyroplasty has become a primary surgical option for voice restoration in patients with glottal incompetency. Type 1 thyroplasty is usually performed in daycare setting requiring patient co-operation under local anaesthesia to test phonation intra-operatively, allowing accurate and adjustable cord medialisation whilst preserving the mucosal wave of the vocal fold.

Gore-Tex ribbon has become a popular implant material in Type 1 thyroplasty, placed with ease through a window created in the thyroid cartilage. ${ }^{1}$ The flexibility of the ribbon allows the degree of medialisation to be distributed differentially along the length of the vocal fold which can be adjusted intra-operatively without involving the removal of the entire prosthesis. The pliability of the material

Corresponding author:

Marina Mat Baki

Department of Otorhinolaryngology

Head and Neck Surgery

Universiti Kebangsaan Malaysia Medical Centre

Jalan Yaacob Latiff, Cheras

56000 Kuala Lumpur, Malaysia.

Email: marinamatbaki@ppukm.ukm.edu.my

Tel: 019-6534308 made it a more natural implant for vocal cord augmentation as it permits contouring of the surrounding tissue.

In Malaysia, not many centres offer the expertise of Type 1 medialisation thyroplasty using Gore-Tex ribbon and to the authors knowledge our centre has the largest number of cases whom had underwent such a procedure. Thus this study is of importance in reviewing the outcome of Gore-Tex Type 1 medialisation thyroplasty in the Malaysian population.

\section{OBJECTIVE}

To review the aetiology of unilateral vocal cord palsy, and the outcome of medialisation thyroplasty using Gore-Tex ribbon implant as the sole primary treatment for unilateral vocal cord palsy in a tertiary referral centre.

\section{MATERIALS AND METHODS}

\section{Subjects}

This is a retrospective review of patients who were referred to the Otorhinolaryngology Department of Universiti Kebangsaan Malaysia Medical Centre and subsequently underwent Gore-Tex medialisation thyroplasty for unilateral vocal cord palsy from 2000 to 2007. Medical records were analysed and data of demographic details, presenting symptoms and the aetiology of the unilateral vocal cord paralysis were collected. The follow-up period was between 2 weeks and 34 months. 


\section{Patient self-evaluation}

The patient has the unique advantage of knowing the quality of his or her premorbid voice. In an attempt to quantify vocal improvement following surgery, the authors used a visual analogue score (VAS) system which rated the voice quality ranging from 1 (aphonia) to 10 (normal voice). The visual analogue scores were obtained before and after surgery. A visual analogue scoring of greater than 5 (VAS > 5) achieved by the patient post-operatively was taken to denote good surgical outcome.

\section{Surgical technique}

Each procedure was performed by the same primary surgeon under local anaesthesia. Significant surgical aspects performed are summarised as follows:

Following a transverse skin crease incision made over the thyroid cartilage and elevation of the platysma, the strap muscles attached to the ipsilateral thyroid lamina is released. Having elevated the outer perichondrium, a window is created using a cutting size 4.0 burr at the estimated level of vocal fold, halfway between the thyroid notch and lower border of thyroid cartilage. The thyroid window is routinely performed above the thyroid tubercle in an ovoid shape with its longest axis measuring 6 to $7 \mathrm{~mm}$. Its distance from the midline ranges 13 to $17 \mathrm{~mm}$. The intact inner perichondrium is then elevated anteriorly, inferiorly and posteriorly creating pockets in the paraglottic space. The Gore-Tex ribbon implant is then inserted into these pockets and the adequacy of medialisation is determined by the patient's phonatory ability to count $1-10$. The wound is closed in layers after haemostasis is secured. There is normally no indication for wound drain postoperatively.

\section{Statistical analysis}

Both pre and post-operative visual analogue scores (VAS) were analysed using Wilcoxon signed ranks and Spearman nonparametric correlation tests to look at their strength of association.

\section{RESULTS}

Of the 50 cases of vocal cord palsy operated during the study period, there were 39 retrievable medical records. Of these, 37 patients were with unilateral vocal cord palsy. There were $18(48.64 \%)$ males and 19 (51.35\%) females with age ranging from 19 to 81 years (mean of 48.7 years). The racial distribution is mainly composed of Malays (59.46\%) with Chinese (21.62\%) and Indians (18.92\%) constitute the remainder. In terms of laterality of vocal cord involvement, 6 $(16.22 \%)$ patients had right sided palsy and 31 (83.78\%) had left sided vocal cord palsy.
Table 1: Aetiology of unilateral vocal cord palsy

\begin{tabular}{ll}
\hline Aetiology & $\begin{array}{l}\text { No of } \\
\text { cases (\%) }\end{array}$ \\
\hline Surgery & $16(43.2 \%)$ \\
Thyroidectomy & $1(2.7 \%)$ \\
Cardiothoracic surgery - & \\
dissecting thoracic aneurysm & $1(2.7 \%)$ \\
RND for parotid carcinoma & $1(2.7 \%)$ \\
Carotid body tumour excision & $1(2.7 \%)$ \\
Excision mediastinal teratoma & $1(2.7 \%)$ \\
Excision of acoustic neuroma & $1(2.7 \%)$ \\
pamma-knife excision of & \\
Idiopathic & $5(13.5 \%)$ \\
Aortic arch aneurysm & $2(5.4 \%)$ \\
Vagal schwannoma & $2(5.4 \%)$ \\
Congenital & $1(2.7 \%)$ \\
Lung carcinoma & $2(5.4 \%)$ \\
Pulmonary tuberculosis & $1(2.7 \%)$ \\
Cerebrovascular accident & $1(2.7 \%)$ \\
Post radiotherapy for nasopharyngeal \\
carcinoma
\end{tabular}

RND = Radical Neck Dissection

The aetiological factors of vocal cord palsy are given in Table 1. Thyroidectomy was the predominant cause of vocal cord palsy in our series. Thirteen out of 16 patients had underwent thyroidectomy for benign thyroid disease whilst the remaining three patients were for thyroid cancer.

There were four cases where palsy had resulted from high vagal nerve lesion; cerebrovascular accident, post radiotherapy for nasopharyngeal carcinoma with multiple cranial nerve palsies, excision of acoustic neuroma with resultant multiple lower cranial nerves palsies and gamma-knife surgical sequelae of a recurrent petrous temporal haemangioma excision respectively.

Hoarseness is a major presenting feature seen in this series; $35.1 \%$ of our patients had hoarseness as the sole presenting symptom whereas $24.3 \%$ had aspiration as an additional component to their hoarseness. Other presenting symptoms include vocal fatigue, vocal pain, dysphagia and foreign body sensation.

There were two significant postoperative airway complications observed in our series of patients (Table 2). 


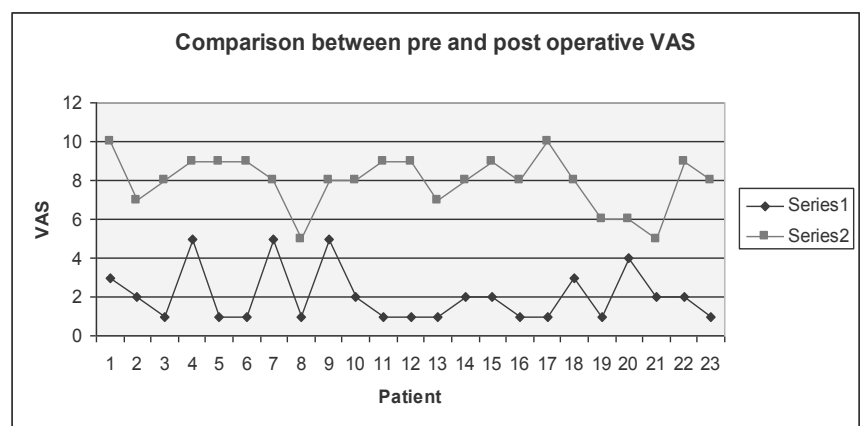

Figure 1: Comparison between pre and post operative VAS

Series 1 = pre operative visual analogue score (VAS)

Series 2 = post operative visual analogue score (VAS)

Twenty three out of $37(62.16 \%)$ visual analogue scores (VAS) were available for analysis (Figure 1). The authors have taken into account that a visual analogue scoring of greater than 5 achieved postoperatively is considered as good postoperative outcome. This was observed in $91.3 \%(n=21)$ patients. Statistical analysis indicated that there is a significant difference in the pre and post-operative VAS $(p<0.001)$. The preoperative VAS has an influence on postoperative VAS as demonstrated by Spearman test for nonparametric correlation (Spearman rho 0.718 at $p<0.0001)$. However age and gender did not have any significant associations with VAS.

We observed that in the three out of four cases whereby the vocal cord palsy is secondary to high vagal nerve lesions and whom presented with hoarseness and aspiration, there was persistent aspiration despite achieving improvement in their VAS postoperatively.

At 6 months follow up, only three out of 37 patients had persistent aspiration following medialisation thyroplasty using Gore-Tex implant which occurred in patients with high vagal nerve lesions. Only one of these patients had worsening aspiration requiring revision surgery and the others recovered with swallowing rehabilitation. The effectiveness in improving aspiration in this series was $91.89 \%$ at 6 months follow up.

In our series, two patients (5.4\%) underwent revision medialisation thyroplasty using Gore-Tex implant. The indications for revision were gradual aphonia 5 months after the initial thyroplasty and worsening aspiration occurring three years following the initial surgery respectively.

\section{DISCUSSION}

Voice breathiness and aspiration represent the main symptoms of unilateral vocal cord palsy which can be very disabling for patients. These symptoms arise as a result of failure of the contralateral normal vocal cord to approximate the paralysed vocal cord thus precluding adequate glottic closure. The management of glottic insufficiency arising from unilateral vocal cord palsy encompasses a wide variety of surgical techniques including vocal cord injection, transcartilaginous augmentation (medialisation thyroplasty) and arytenoid adduction. ${ }^{1}$ The common aim shared by all the varied surgical methods is to achieve optimum glottic closure by positioning the paralysed vocal cord in a median position allowing contact with the normal vocal cord. Since Isshiki's introduction of his surgical technique, type 1 medialisation thyroplasty remains the surgical procedure of choice widely used in the treatment of glottic insufficiency. In 1998, Mc Culloch and Hoffman introduced the use of expanded polytetrafluoroethylene (Gore-Tex) implant in medialisation thyroplasty.

The technique is potentially reversible as the implant can be removed easily in the face of revision surgery, well tolerated by the patients, gives stable and satisfactory voice outcome as well as safe. ${ }^{2}$ In the present study, all operations were conducted using local anaesthesia. If the voice quality is suboptimal, the Gore-Tex ribbon implant was taken out, reinserted and repositioned until the best voice is obtained thus fine tuning the voice outcome. The operation is suitable for patients with high anaesthetic risk as it only requires local anaesthesia. As with other centres, thyroplasty is a daycare surgical procedure at Universiti Kebangsaan Malaysia Medical Centre.

Table 2: Airway complication of Gore-Tex medialisation thyroplasty for unilateral vocal cord palsy

\begin{tabular}{lllll}
\hline $\begin{array}{l}\text { Patient } \\
\text { no }\end{array}$ & $\begin{array}{l}\text { Age / } \\
\text { Gender }\end{array}$ & $\begin{array}{l}\text { Postopera- } \\
\text { tive onset } \\
\text { (hours) }\end{array}$ & $\begin{array}{l}\text { Causative } \\
\text { factor }\end{array}$ & Management \\
\hline 3 & $\begin{array}{l}49 \mathrm{y} / \\
\mathrm{F}\end{array}$ & $24 \mathrm{~h}$ & $\begin{array}{l}\text { Supraglottic } \\
\text { oedema }\end{array}$ & $\begin{array}{l}\text { Tracheosto- } \\
\text { my }\end{array}$ \\
12 & $\begin{array}{l}80 \mathrm{y} / \\
\mathrm{M}\end{array}$ & $5 \mathrm{~h}$ & Haematoma & $\begin{array}{l}\text { Haematoma } \\
\text { evacuation }\end{array}$ \\
\hline
\end{tabular}

Although relatively few, complications following type 1 medialisation thyroplasty can occur and the spectrum of complications reported in the literature included haematoma, wound infection, implant extrusion (ranging from 6.67\%-8.65\%) ) $^{2,3}$ and dislodgement, and airway obstruction $(10 \%){ }^{3}$

In this series postoperative complication of airway compromise presenting as stridor was observed in 2 cases (5.4\%). Patient 3 (Table 2) developed supraglottic oedema which required emergent tracheostomy at postoperative day 1 . Aryepiglottic fold haematoma was the culprit in patient 12 (Table 2 ) occurring five hours after surgery. Our centre has adopted the policy of hospital admission for close postoperative monitoring in those patients regarded as high anaesthetic risk and the elderly.

In contrast to the decreasing trend in unilateral vocal cord palsy caused by thyroid surgery reported 
elsewhere from $28 \%$ in 1970 to $4.1 \%$ as reported by Ramadan et al in $1998,{ }^{4}$ the major aetiological factor in our series remained thyroidectomy (43.2\%). Recurrent laryngeal nerve palsy (RLNP) is a recognised complication after thyroid surgery, more so if the surgery is indicated for malignancy. Nevertheless recurrent laryngeal nerve palsy is seen in $7.3 \%$ of thyroidectomy performed for benign

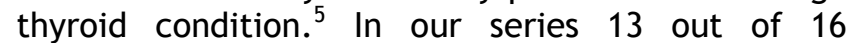
(81.3\%) cases of thyroidectomy related unilateral vocal cord palsy (UVCP) were for benign condition outweighing the three cases of thyroidectomy for papillary thyroid carcinoma (18.7\%). We postulate that this may be due to more thyroidectomies were performed for benign diseases of the thyroid compared to malignant thyroid disease. Additionally, intraoperative nerve monitoring has not yet gain popularity in Malaysia.

When the recurrent laryngeal nerve (RLN) is injured, spontaneous recovery of function can be anticipated up to one year after the initial insult providing that the nerve remains intact. However once the nerve is severed such recovery will not be possible. The incidence of temporary RLNP post thyroidectomy has been reported as $9.8 \%$ and $2.3 \%$ for permanent RLNP. ${ }^{6}$ Based on this consensus, follow-up period up to one year to assess and evaluate RLNP has been recommended before committing patients to medialisation thyroplasty. ${ }^{6}$ Other important deciding factor in the timing for the procedure is the cause of the resultant vocal cord palsy. ${ }^{1}$

Thus early Gore-Tex medialisation thyroplasty is adopted by the authors in our centre for those patients in whom RLNP is secondary to malignancy (be it thyroid or intrathoracic), or in whom voice breathiness or vocal fatiguability is most distressing, or associated with aspiration. This in part explains the wide period between the initial thyroidectomy and Gore-Tex medialisation thyroplasty observed in the series ranging between 4 months to 14 years. The much delayed period between thyroidectomy and medialisation thyroplasty may also be a reflection of the level of awareness in our population, patients and medical professionals alike, as to the availability of surgical options and expertise to address the issue of symptomatic RLN related vocal cord palsy.

Satisfactory results of medialisation thyroplasty have been reported by numerous investigators. ${ }^{7-13}$ In the present series the assessment of vocal outcome was made subjectively by the patient using a visual analogue score. With a score of above 5 taken to denote good outcome $65.2 \%(n=15)$ of our patients had achieved significant improvement in their voice visual analogue score (VAS) following Gore-Tex medialisation thyroplasty. Not only the difference between pre and post-operative VAS is statistically significant $(p<0.001)$ but there is a linear correlation between them with preoperative VAS having an influence on the postoperative VAS. Thus a patient with severe dysphonia may not attain immense improvement in his voice in comparison to a patient with a less severe degree of dysphonia.

We observed that patients $(n=4)$ with high vagal nerve lesion despite achieving good vocal outcome the accompanying aspiration persisted. In high vagal nerve lesion the larynx become incompetent as a result of the disruption of both sensory and motor pathways leaving the patient vulnerable to aspiration. The aetiologies of the high vagal nerve lesions in this series included skull base tumours $(n=2)$, cerebrovascular accident $(n=1)$ and radiotherapy induced cranial palsies for nasopharyngeal carcinoma $(n=1)$. Medialisation thyroplasty alone is insufficient to address the problem of aspiration necessitating adjunct arytenoid adduction to further improve the posterior glottic closure which cannot be achieved by thyroplasty alone. ${ }^{14-15}$

The indications for revision thyroplasty fall into two main groups, complications and glottal closure problems. $^{16}$ Complications include extruded or displaced implants. The commonest glottal closure problem is under correction, others include anterior overcorrection and a persistent posterior glottal gap. Our series observed two patients required revision thyroplasty indicated by aphonia (undercorrection) and worsening aspiration respectively however there was no cases of implant extrusion in the present series.

Our main limitation is in the objective assessment pre and post-operatively. We relied mainly on endoscopic evaluation of the vocal folds and the glottic gap and with visual analogue score (VAS) for subjective vocal outcome measure. The authors look forward to greater efforts toward standardising outcome evaluation with better objective measuring tools.

\section{CONCLUSION}

Gore-Tex medialisation thyroplasty alone as the phonosurgical method for the treatment of unilateral vocal cord palsy is effective in addressing dysphonia in our study population. However it is not sufficient in addressing accompanying aspiration in cases of high vagal lesions. Thyroid surgery still carries a risk of vocal cord palsy and it remains pertinent that not only the patient be counseled regarding the accompanying risk but also of thyroplasty as a treatment option should the complication occur.

\section{REFERENCES}

1. Mahieu HF. Practical application of laryngeal framework surgery. Otolarynygol Clin North Am 2006; 39:55-75.

2. McCulloch TM, Hoffman HT. Medialization laryngplasty with expanded polytetrafluoroethylene. Ann Otol Rhinol Laryngol 1998;107:427-32. 
3. Cotter CS, Avidano MA, Crary MA, Cassisi NJ, Gorham MM. Laryngeal complications after type 1 thyroplasty. Otolaryngol Head Neck Surg 1995;113:671-3.

4. Tucker HM, Wanamaker J, Trott M, Hicks D. Complications of laryngeal framework surgery (phonosurgery). Laryngoscope 1993;103:525-8.

5. Ramadan HH, Wax MK, Avery S. Outcome and changing cause of unilateral vocal cord paralysis. Otolaryngol Head Neck Surg 1998;18:199-202.

6. Yumoto E, Minoda R, Hyodo M, Yamagata T. Causes of recurrent laryngeal nerve paralysis. Auris Nasus Larynx 2002;29:41-5.

7. Jeannon JP, Orabi AA, Bruch GA, Abdalsalam HA, Simo R. Diagnosis of recurrent laryngeal nerve palsy after thyroidectomy: a systematic review. Int J Clin Pract 2009;63:624-9. 